\title{
Residual dust charges in discharge afterglow
}

\author{
L. Couëdel, ${ }^{*}$ M. Mikikian, and L. Boufendi \\ GREMI (Groupe de Recherches sur l'Énergétique des Milieux Ionisés), \\ CNRS/Université d'Orléans, 14 rue d'Issoudun, 45067 Orléans Cedex 2, FRANCE \\ A. A. Samarian \\ School of Physics A28, University of Sydney, NSW 2006, AUSTRALIA
}

\begin{abstract}
An on-ground measurement of dust particle residual charges in the afterglow of a dusty plasma was performed in a rf discharge. An upward thermophoretic force was used to balance the gravitational force. It was found that positively-charged, negatively-charged and neutral dust particles coexisted for more than one minute after the discharge was switched off. The mean residual charge for $200 \mathrm{~nm}$ radius particles was measured. The dust particle mean charge is about $-5 e$ at pressure of $1.2 \mathrm{mbar}$ and about $-3 e$ at pressure of 0.4 mbar.
\end{abstract}

PACS numbers: 52.27.Lw

\section{INTRODUCTION}

Dusty or complex plasmas are partially ionized gas composed of neutral species, ions, electrons and charged dust particles. In laboratory experiments, these particles can be either injected or grown directly in the plasma. Injected dust particles are usually micron-size particles. Due to their mass, they are confined near the bottom electrode where the electric force counterbalances gravity. Microgravity condition is necessary to study dust clouds of micrometer size particles filling the whole plasma chamber [1]. In laboratory, dense clouds of submicron particles light enough to completely fill the gap between the electrodes can be obtained using reactive gases such as silane $[2,3]$ or using a target sputtered with ions from plasma [4-7]

Dust particle charge is a key parameter in complex plasma. It determines the interaction between a dust particle and electrons, ions, its neighboring dust particles, and electric field. The determination of the dust particle charge is so one of the basic problems in any complex plasma experiments. The knowledge of dust charge will allow us to understand the basic properties of dusty plasma, particle dynamics in dust clouds, and methods to manipulate the particles.

The particle charge controls the dust dynamics both in laboratory and technological plasma reactors, and also in space plasmas. Thus one of the main dusty plasma challenges is to understand the dust charging in a wide range of experimental conditions, which simulates industrial and space plasmas. For example, in most industrial processes in the microelectronics industry which uses silane as the reactive gas, dust contamination is a vital problem [8]. The dust dynamics and coagulation in space plasma are also phenomena governed by dust particle charges $[9,10]$.

There are many publications reporting on the investigation of dust charging in discharge plasma, see [11-18] and references therein. However there are only few papers devoted to dust charging, or decharging to be more specific, in the discharge afterglow. In [19] a diffusion of

*Electronic address: lenaic.couedel@univ-orleans.fr fine dust particles $(8-50 \mathrm{~nm})$ in the afterglow of a dusty plasma has been studied. From the afterglow decay of dust number density the charged fraction of particle have been measured. It was found that some particles carried a very small residual charge and some were neutral. A model has been proposed to explain charges on particles in the late afterglow of a dusty plasma. An observation of decay, or "decharging", of a rf plasma with dust particles after switching off discharge power was reported in [20]. Experiments were performed in the PKE-Nefedov reactor [1] under microgravity conditions on board International Space Station. An existence of negative residual charges has been shown. A simple theoretical model that describes data obtained was proposed. The residual charges were attributed to the presence of an excitation electric field that was used for dust charge measurements. Limitation of the Space Station experiment routine could not prove this hypotheses. Theoretical predictions on an effect of rf plasma parameters on residual charges has neither been verified.

So any new experimental evidence of residual dust charges in afterglow plasma will lead to a better understanding in the decharging of complex plasma. Indeed the value and nature of residual charges after plasma extinction is of great importance. The dust particle charge in afterglow plasma could induce problem in future single-electron devices where a residual charge attached on deposited nanocrystals would be the origin of dysfunction. It could make easier industrial plasma processing reactor decontamination thanks to the use of specially designed electric field. The residual charges on dust particles in fusion reactors (such as ITER) also can make the cleaning process much easier.

In this paper, we report the first on-ground experiment on the residual charge measurement of dust particles after decay of a dusty plasma. The experiment was performed in the PKE-Nefedov reactor where the dust particles were physically grown in the plasma. Temperature gradient was introduced in the chamber to create an upward thermophoretic force to balance gravity. The residual charges were determined from analysis of dust oscillations, which were excited by applying sinusoidal bias to the bottom electrode. It was found coexistence of positively and negatively charged dust particles as well as non-charged ones for more than one minute after the discharge was switched off. The residual charges for $200 \mathrm{~nm}$ radius par- 


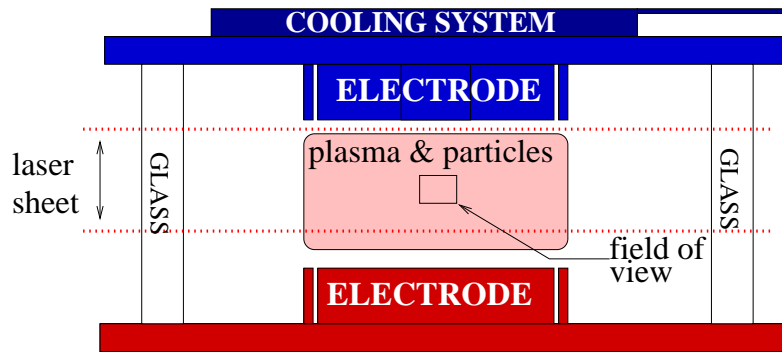

FIG. 1: (Color online) Schematic of the experimental apparatus

ticles have been measured for two different pressures. It was revealed that dust particles kept the residual charges only when the discharge was abruptly switched off. In the case when the discharge power is decreased slowly until the plasma disappeared, there was no residual charge on dust particles. It was also shown that the presence of the low frequency excitation electric field did not play any role in keeping the charge on dust particles in the afterglow plasma.

The article is organized as following way. Section II is devoted to general description of experimental set-up. In section III we analyze the forces acting on dust particles, paying most attention to the thermophoretic force. In section IV, we discuss a procedure of residual charge measurement and present experimental data obtained, in section $\mathrm{V}$ we analyze discharging of dust particle in afterglow plasma, and section VI is conclusion.

\section{EXPERIMENTAL SETUP}

The work presented here is performed in the PKENefedov (Plasma Kristall Experiment) chamber designed for microgravity experiments [1]. It is a rf discharge operating in push-pull excitation mode. It consists of $4 \mathrm{~cm}$ diameter parallel electrodes separated by $3 \mathrm{~cm}$. The injected power varies in the range $0-4 W$. Dust particles are grown in an argon plasma $(0.2-2$ mbar $)$ from a sputtered polymer layer deposited on the electrodes and coming from previously injected dust particles (3.4 $\mu \mathrm{m}$, melamine formaldehyde). A detailed description of this experiment and previous results are presented in Ref $[1,4,5]$.

For the study concerning residual charges, the top electrode was cooled (Fig. 1). An upward thermophoretic force was applied to dust particles in order to counterbalance gravity [21] when the plasma is off. To study particle charges, a sinusoidal voltage produced by a function generator with amplitude $\pm 30 \mathrm{~V}$ and frequency of $1 \mathrm{~Hz}$ was applied to the bottom electrode. Induced low frequency sinusoidal electric field $\boldsymbol{E}(\boldsymbol{r}, t)$ generated dust oscillations if they kept a residual electric charge. A thin laser sheet perpendicular to the electrodes illuminates dust particles and the scattered light is recorded at $90^{\circ}$ with standard charge coupled device (CCD) cameras with 25 images per second. Video signals were transferred to a computer via a frame-grabber card with 8 bit gray scale and $560 \times 700$ pixel resolution. In order to avoid edge effect, a field of view over $8.53 \times 5.50 \mathrm{~mm}^{2}$ restrained to the center of the chamber was used for residual charge measurement. By superimposition of video frames particles trajectories have been obtained. The coordinates of the particles were measured in each third frame. The amplitude of the oscillations was figured out from the measured particle positions. Absolute values for the oscillation amplitude were obtained by scaling the picture pixels to the known size of the field of view.

\section{FORCES ACTING ON DUST PARTICLES}

Dust particles in laboratory plasmas are subjected to various forces that confine them in the plasma or drag them out (to the walls or pump outlet)[8]: the confining electrostatic force, the gravity, the thermophoretic force, the ion drag force and the neutral drag force.

After the discharge is switched off, the forces still acting on dust particles are the gravity $F_{g}$, the thermophoretic force $F_{T}$, the neutral drag force $F_{d n}$ and, if dust particles keep residual charges, the electric force $F_{E}$ due to the electric field induced by the sinusoidal voltage applied to the lower electrode. In this experiment the thermophoretic force balanced the gravity force, and electrostatic force was the reason for the dust oscillations while the neutral drag force damp the last ones.

Expression for the gravity force is:

$$
\boldsymbol{F}_{\boldsymbol{g}}=\frac{4}{3} \pi r_{d}^{3} \cdot \rho \boldsymbol{g}
$$

where $\boldsymbol{g}$ is the gravity acceleration, $r_{d}$ is the dust particle radius, and $\rho$ its mass density.

The neutral drag force was taken as [22]:

$$
\boldsymbol{F}_{\boldsymbol{d} \boldsymbol{n}}=-\frac{8}{3} \sqrt{2 \pi} r_{d}^{2} m_{n} n_{n} v_{T n}\left(1+\alpha_{a c} \frac{\pi}{8}\right)\left(\mathbf{v}_{\mathbf{d}}-\mathbf{v}_{\mathbf{n}}\right)
$$

where $m_{n}$ is the neutral atom mass, $n_{n}$ the neutral atom density, $v_{T n}=\sqrt{8 k_{B} T_{n} / \pi m_{n}}$ the thermal speed with $T_{n}=T$ the neutral gas temperature, $k_{B}$ the Boltzmann constant, $\alpha_{a c}$ the accomodation coefficient, $\mathbf{v}_{\mathbf{d}}$ dust particle speed and $\mathbf{v}_{\mathbf{n}}$ a mean speed of neutral atoms. In our experiment $\mathbf{v}_{\mathbf{n}}$ can be taken as 0 because there is no gas flow.

The electric force can be written as

$$
\boldsymbol{F}_{\boldsymbol{E}}(\boldsymbol{r}, t)=Q_{d} \boldsymbol{E}(\boldsymbol{r}, t)
$$

where $Q_{d}$ is the dust particle electric charge and $\boldsymbol{E}(\boldsymbol{r}, t)$ is the electric field between the electrodes after the plasma is switched off. In the late afterglow the plasma density is very small and $\boldsymbol{E}(\boldsymbol{r}, t)$ can be approximated by the vacuum field above a charged disk. Taking into account size of the camera field of view, size of the electrode and the fact that camera was directed to the region near center of the chamber, we are interested in the electric field vertical component $\boldsymbol{E}(\boldsymbol{r}, t) \approx E_{z}(z, t) \vec{e}_{z} \equiv E(z, t) \vec{e}_{z}$ only. The calculated value of $E(z, t)$ at the central axis is presented in Fig.2

The expression for the thermophoretic force must be choosen carefully. Indeed, it depends strongly on Knudsen number $K n=l / r_{d}$ [23] where $l$ is the mean free 


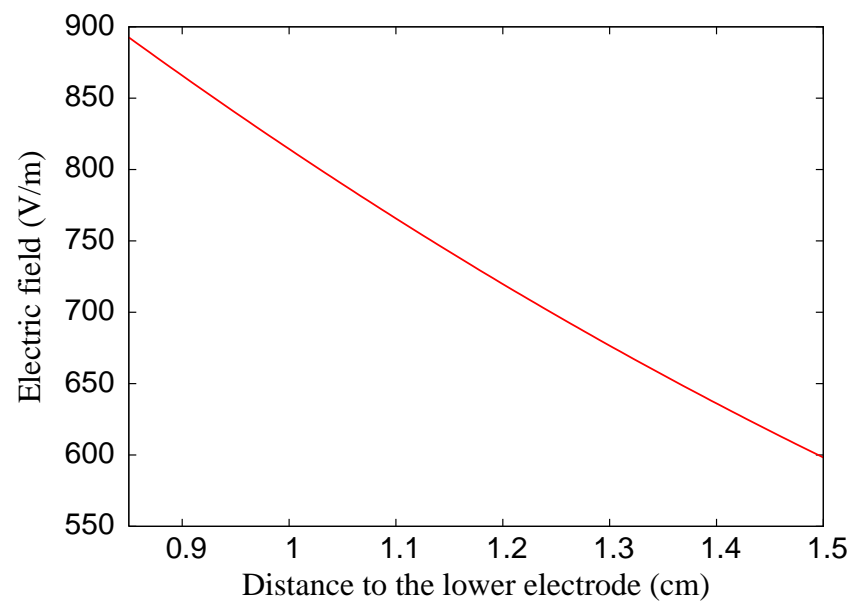

FIG. 2: (Color online) Vertical component of the electric field in the camera field of view

path of buffer gas species. In our experiment, we work at an operating pressure around 1 mbar. In a previous paper [4], the size of grown dust particles was reported between $200 \mathrm{~nm}$ and $800 \mathrm{~nm}$. It gives, using results from Varney [24] for an atom-atom cross section, Knudsen number $250<K n<1000$. Consequently we operate in free molecular regime where a dust particule is similar to a very large molecule. Many theories have been developped [23, 25-28] and used [21, 23, 29] for thermophoresis in the free molecular regime. The most commonly used equation is the Waldmann equation [28] which has been verified experimentally $[30,31]$ :

$$
\boldsymbol{F}_{\boldsymbol{T}}=-\frac{32}{15} r_{d}^{2} \frac{k_{t r}}{v_{T n}} \nabla T
$$

where $\nabla T$ is the temperature gradient in the gas, and $k_{t r}$ the translational part of the thermal conductivity given for a monoatomic gas by [32]:

$$
k_{t r}=\frac{15 k_{B}}{4 m_{n}} \mu_{r e f}\left(\frac{T}{T_{r e f}}\right)^{\nu}
$$

where $\mu_{\text {ref }}$ is the reference viscosity at reference temperature $T_{r e f}=273 \mathrm{~K}$ and the exponent $\nu$ results from a best fit of experimental viscosity near the reference temperature. For argon, $\mu_{\text {ref }}=2.117 \cdot 10^{-5} \mathrm{~Pa} \cdot s$ and $\nu=0.81$ [32].

Another important effect that must be considered in the estimation of thermophoretic force is an influence of the finite volume of gas. If pressure is low enough, gas mean free path can become comparable to a length scale of experimental apparatus and the gas can no longer be treated as a continuous medium. Under such condition, an additional Knudsen number must be added [23] $K n_{L}=l / L$ where $L$ is the length scale of the reactor. In this experiment, the length between electrodes is $L=3 \mathrm{~cm}$ giving $K n_{L} \sim 5 \cdot 10^{-3}$ which means the gas can be considered as a continuous medium.

Temperature gradient between the electrodes was calculated by FEMLAB ${ }^{\circledR}$ code (steady state analysis of heat transfer through convection and conduction with heat flux, convective and temperature boundary condi-

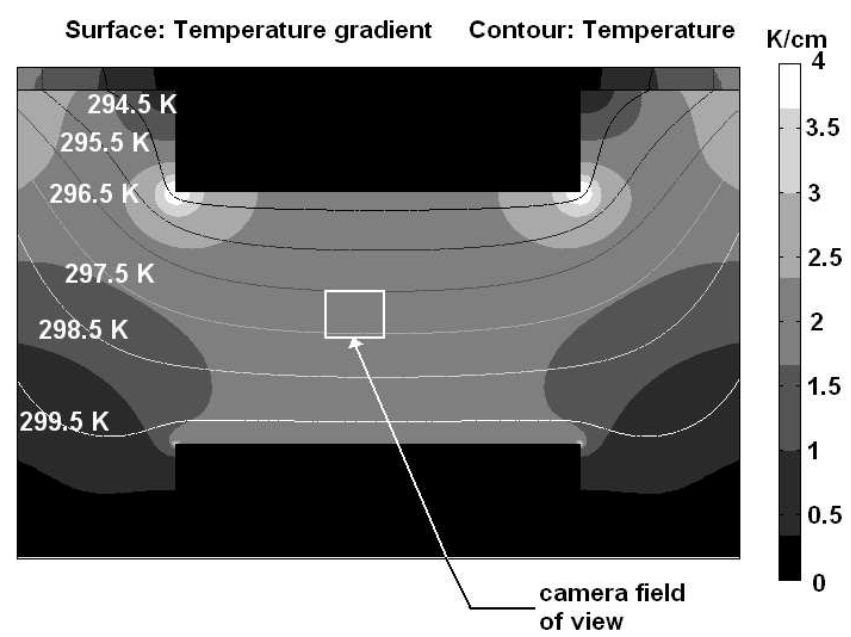

FIG. 3: Temperature profile and gradient

tions using Lagrange-Quadratic element). The temperatures on the electrodes were measured by thermocouple and used as boundary conditions for the problem. The countour-plot obtained is presented in Fig.3. It shows that the vertical component of the temperature gradient is constant near reactor center. The value of temperature gradient is about $2 \mathrm{~K} / \mathrm{cm}$ for our experiment. There is also a small horizontal component of the temperature gradient. This is the reason for particle drift in horizontal direction. Such drift allowed us to resolve particle trajectories (see next section) and made particle charge measurement more convenient.

\section{EXPERIMENTAL PROCEDURE AND RESULTS}

In this section, dust particle size estimation and residual charge measurement are presented. The residual charge measurements have been performed by the following routine. First the chamber was pumped down to lowest possible pressure (base pressure $\sim 2 \cdot 10^{-6} \mathrm{mbar}$ ) and the cooling system was turned on. After this, argon was injected up to the operating pressure, the discharge was started and particles were grown, forming familiar structures such as a void ( see for example [5]). Then, the discharge was switched off and bottom electrode was biased by sinusoidal voltage.

In afterglow plasma, dynamics of dust particles is determined by temperature gradient and excitation electric field. Fig. 4 presents superimposition of images taken after discharge had been switched off. There are two different types of motion observable. Dust particles drift upwards, downwards and to the side due to existing temperature gradients and they oscillate due to electrostatic force. It is obvious that thermophoretic force acts on any dust particle in chamber, while the electrostatic force acts only if particles have charge in afterglow. Thus presence of oscillating particles (see Fig.4) clearly indicates that dust particles do have residual charges after the discharge has been switched off. Dust particles oscillated in opposite phases as well as non-oscillating dust grains 


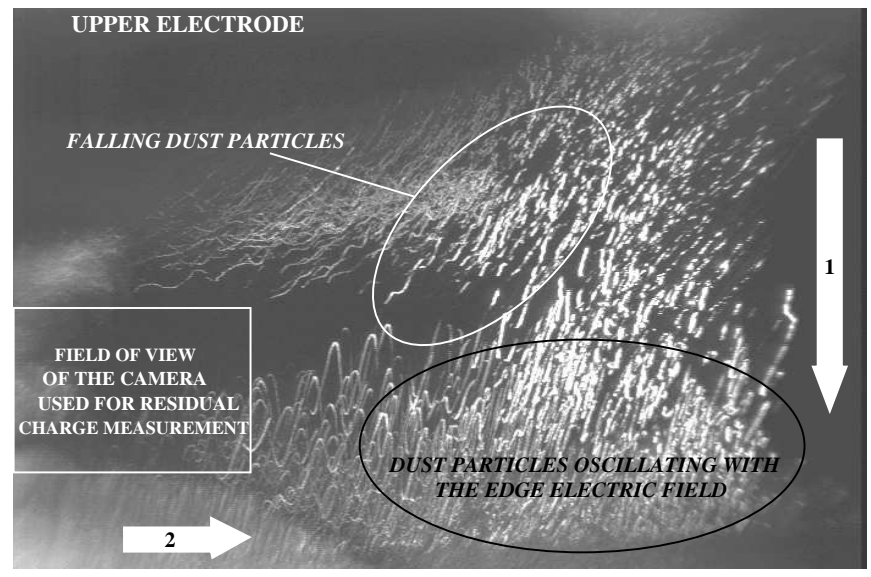

FIG. 4: Superimposition of video frames taken with a large field of view CCD camera. Arrows 1 and 2 represent respectively the vertical and the horizontal components of the temperature gradient. Edge effects as well as falling dust particles can be seen.

have been observed indicating that negatively charged, positively charged and non-charged dust particles coexist after plasma extinction.

It worth to mention that in order to observe dust oscillations the discharge must be switched off abruptly. It was shown that if the power was decreased slowly until the plasma disappears there are no residual charges (no oscillations of dust particles in the sinusoidal electric field were observed). Another interesting fact is that residual charge on dust particles has long relaxation time and does not depend on time when the excitation electric field was applied to. Dust oscillations were observed for more than one minute after plasma extinction and in both cases when the function generator was switched on during the disharge or few seconds after the discharge is turned off.

As it can be seen in Fig.4, there are dust particles falling after the discharge is switched off. These particles are too big to be sustained by the thermophoretic force. Other particles are horizontally drift at constant height, this means for these particles gravity force is balanced by thermophoretic force. These particles have been used to measure residual charges. It is clear from Fig.4, that use of a large field of view camera gives us nice overall picture of decaying dusty plasma but it is not suitable for residual charge measurement because edge effects can not be neglected. Thus the camera with the small field of view was used for the charge measurement (see Fig.4). The superimposition of images from this camera is presented in Fig.5. These images give us clear track of the dust oscillations so dust grain trajectories can be reconstructed (Fig.6).

\section{A. Size and mass of levitating dust grains in discharge afterglow}

Particle size (mass) and residual charge measurement are strongly related in this experiment. Charge, size and mass of dust particles have so to be determined. Considering that dust particles levitating in reactor at

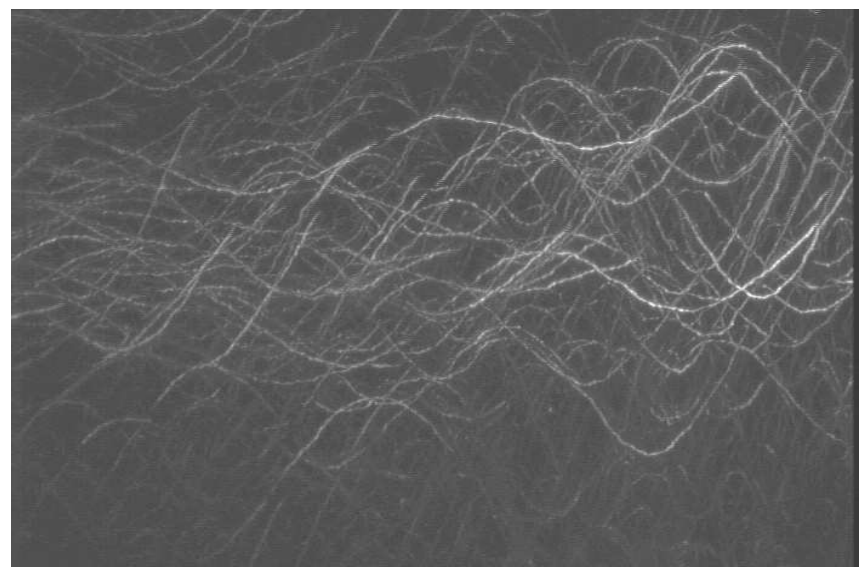

FIG. 5: Superimposition of video frames 10 seconds after plasma extinction. Dust particle oscillations can clearly be seen. The temperature gradient has a slight horizontal component. Therefore, oscillations are in the $2 \mathrm{D}$ laser plane.

a constant height after plasma extinction are the ones for which the gravity is exactly balanced by the thermophoretic force, the dust particle radius can be found using Eq.1 and Eq.4:

$$
r_{d}=-\frac{8}{5 \pi \rho g} \frac{k_{t r}}{v_{t h}} \nabla T
$$

Dust grains are supposed to be spherical and mainly made of carbon (sputtering of a carbonaceous polymer material) [4] so that the mass $m_{d}$ can be deduced from Eq.6:

$$
m_{d}=\frac{4}{3} \pi r_{d}^{3} \cdot \rho
$$

where $\rho$ is the mass density of graphite. In our experimental condition, the radius of levitating dust grains is estimated to $r_{d} \simeq 190 \mathrm{~nm}$ and their mass is $m_{d} \simeq$ $6.5 \cdot 10^{-17} \mathrm{~kg}$.

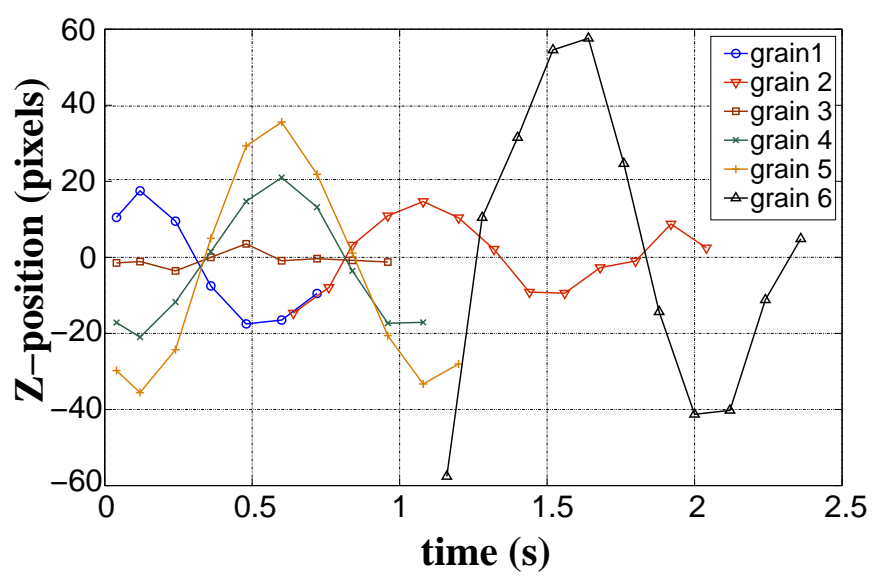

FIG. 6: (Color online) Oscillation of 6 dust grains 10 seconds after plasma extinction. Non oscillating dust grain and opposite phase oscillations are observed. 


\begin{tabular}{|c|c|c|}
\hline & $P=1.2 \mathrm{mbar}$ & $P=0.4 \mathrm{mbar}$ \\
\hline \hline$\nabla T$ & $-190 \mathrm{~K} \cdot \mathrm{m}^{-1}$ & $-177 \mathrm{~K} \cdot \mathrm{m}^{-1}$ \\
\hline$r_{d}$ & $194 \mathrm{~nm}$ & $180 \mathrm{~nm}$ \\
\hline$m_{d}$ & $6.9 \cdot 10^{-17} \mathrm{~kg}$ & $5.4 \cdot 10^{-17} \mathrm{~kg}$ \\
\hline$\gamma$ & $1.36 \cdot 10^{-13} \mathrm{~kg} \cdot \mathrm{s}^{-1}$ & $0.39 \cdot 10^{-13} \mathrm{~kg} \cdot \mathrm{s}^{-1}$ \\
\hline$Q_{d_{\text {res } \max }}$ & $+2 e$ & $+2 e$ \\
\hline$Q_{d_{\text {res }} \min }$ & $-12 e$ & $-4 e$ \\
\hline$Q_{d_{\text {res }} \text { mean }}$ & $-5 e$ & $-3 e$ \\
\hline
\end{tabular}

TABLE I: Measured maximum, minimum and mean dust particle residual charges for two operating pressures.

\section{B. Dust charge measurement}

From the measurement of oscillation amplitude, the residual charge on a dust particle can be obtained. As the gravity is compensated by the thermophoretic force, the equation of motion for one dust particle, neglecting its interactions with other dust particles, can be reduced to:

$$
m_{d} \ddot{z}=F_{E}(z, t)+F_{n d}(\dot{z})
$$

Taking $E(t)=E_{0}\left(z_{\text {mean }}\right) \cos (\omega t)$ (the amplitude of the electric field $E_{0}$ is the one at the mean dust levitation height $z_{\text {mean }}$ ) and using Eq.3, Eq.2 and Eq.8, the dust particle oscillation amplitude $b$ can be obtained [33]:

$$
b\left(\omega, Q_{d}, E_{0}\right)=\frac{Q_{d} E_{0}\left(z_{\text {mean }}\right)}{m_{d} \omega \sqrt{\omega^{2}+4 \gamma^{2} / m_{d}^{2}}}
$$

where $\gamma=(4 / 3) \sqrt{2 \pi} r_{d}^{2} m_{n} n_{n} v_{T n}\left(1+\alpha_{a c}(\pi / 8)\right)$ is the damping coefficient and $\omega=2 \pi f$ where $f$ is the frequency imposed by the function generator. Eq.9 can easily be inversed to derive the dust residual charge:

$$
Q_{d_{\text {res }}}=\frac{m_{d} b\left(\omega, Q_{d}, E_{0}\left(z_{\text {mean }}\right)\right) \omega \sqrt{\omega^{2}+4 \gamma^{2} / m_{d}^{2}}}{E_{0}\left(z_{\text {mean }}\right)}
$$

The sign of the dust particle charge is deduced from the phase of the dust particle oscillation with respect to the excitation electric field. Oscillation amplitudes up to $1.1 \mathrm{~mm}$ have been measured (depending on the operating pressure) and charges from $-12 e$ to $+2 e$ are deduced where $e$ is the elementary charge. It has been found that at high pressure dust particles keep a higher mean residual charge (Tab.I) but uncertainties are of $\sim 2 e$ for each measurement.

\section{DISCUSSION}

The charging (discharging) process of dust particle in a plasma is governed by the contributions of all currents entering (or leaving) the dust surface, involving plasma electron and ion currents, photoemission and thermionic emission currents, etc:

$$
\frac{d Q_{d}}{d t}=\sum I_{a}+\sum I_{l}
$$

where $I_{a}$ and $I_{l}$ are currents absorbed and emitted by the particle (with appropriate sign). In most cases for discharge plasmas we can ignore the emission current and kinetics of the particle charge can be expressed as:

$$
\frac{d Q_{d}}{d t}=J_{i}-J_{e}=-\pi e r_{d}^{2}\left[n_{e} v_{T_{e}} e^{-\varphi}-n_{i} v_{T_{i}}\left(1+\tilde{T}_{e} \varphi\right)\right]
$$

where $J_{e}$ and $J_{i}$ are the fluxes of electrons and ions onto the particle, $v_{T_{i}(e)}=\sqrt{8 k_{B} T_{i(e)} / \pi m_{i(e)}}$ the thermal velocity of ions (electrons), $\tilde{T}_{e}=T_{e} / T_{i}$ is the electron to ion temperature ratio, $n_{i(e)}$ is the density of ions (electrons) and $\varphi=-e Q_{d} / 4 \pi \epsilon_{0} k_{B} r_{d} T_{e}$ is the dimensionless surface potential of a dust particle where $\epsilon_{0}$ is vacuum dielectric permitivity. According to Eq.12, charge on dust particle depends on the electron-ion masses, temperatures and density ratios $m_{e} / m_{i}, n_{e} / n_{i}, T_{e} / T_{i}$. Thus to analyze the decharging of dust particle in afterglow plasma one need to consider the kinetics of plasma decay.

The plasma diffusion loss and electron temperature relaxation determine kinetics of the discharge plasma decay [34]. In presence of dust particles, plasma loss is due to diffusion onto the walls completed by surface recombination on dust particles. Equations for plasma density and electron temperature relaxation are [34, 35]:

$$
\begin{aligned}
\frac{d \tilde{n}}{d t} & =-\frac{\tilde{n}}{\tau_{L}} \\
\frac{d \tilde{T}_{e}}{d t} & =-\frac{\tilde{T}_{e}-1}{\tau_{T}}
\end{aligned}
$$

where $\tilde{n}=n_{i, e} / n_{0}$ with $n_{0}$ the initial plasma density, $\tau_{L}$ the time scale of the plasma loss, and $\tau_{T}$ is the time scale for electron temperature relaxation. The expressions for the time scales are $[34,35]$ :

$$
\begin{aligned}
& \tau_{L}^{-1}=\tau_{D}^{-1}+\tau_{A}^{-1} \\
& \tau_{D}^{-1} \simeq \frac{l_{i n} v_{T_{i}}}{3 \Lambda^{2}}\left(1+\tilde{T}_{e}\right) \equiv \frac{1}{2}\left(1+\tilde{T}_{e}\right) \frac{1}{\tau_{D}^{\infty}} \\
& \tau_{A}^{-1} \simeq \pi r_{d}^{2} n_{d} v_{T_{i}}\left(1+\varphi \tilde{T}_{e}\right) \equiv\left(\frac{1+\varphi \tilde{T}_{e}}{1+\varphi}\right) \frac{1}{\tau_{A}^{\infty}} \\
& \tau_{T}^{-1}=\sqrt{\frac{\pi}{2}} \sqrt{\frac{m_{e}}{m_{i}}} \frac{v_{T_{i}}}{l_{e n}} \sqrt{\tilde{T}_{e}} \equiv \frac{\sqrt{T_{e}}}{\tau_{T}^{\infty}}
\end{aligned}
$$

where $\tau_{D}$ is the ambipolar diffusion time scale onto the walls, $\tau_{A}$ the particle absorption time scale, $l_{i(e) n}$ the mean free path of ion (electron)-neutral collision, $\Lambda$ is the characteristic diffusion length $(\Lambda \sim 1 \mathrm{~cm}$ in this experiment). The $\infty$ exponent stands for the limit at very long time.

For charging time scale lower than plasma decay or temperature relaxation time scales the charge on dust particle is in equilibrium, i.e. ion and electron fluxes balance each other, $\varphi \simeq \varphi_{e q}$ and using Eq.12, $\varphi_{e q}$ is given by:

$$
\frac{n_{e}}{n_{i}} \sqrt{\tilde{T}_{e}} e^{-\varphi_{e q}}=\sqrt{\frac{m_{e}}{m_{i}}}\left(1+\tilde{T}_{e} \varphi_{e q}\right)
$$

In this case, expressions for charge fluctuation and charge fluctuation time scale $\tau_{Q}$ are:

$$
\begin{aligned}
\frac{d Q_{d}}{d t} & \simeq-\frac{Q_{d}-Q_{d e q}}{\tau_{Q}} \\
\tau_{Q}^{-1} & \simeq \frac{v_{T_{i}} r_{d}}{4 \lambda_{i 0}^{2}}\left(1+\varphi_{e q}\right) \tilde{n} \equiv \frac{\tilde{n}}{\tau_{Q}^{0}}
\end{aligned}
$$




\begin{tabular}{|c|c|c|}
\hline & $P=0.4 \mathrm{mbar}$ & $P=1.2 \mathrm{mbar}$ \\
\hline \hline$\tau_{D}^{0}$ & $30 \mu \mathrm{s}$ & $90 \mu \mathrm{s}$ \\
\hline$\tau_{D}^{\infty}$ & $1.5 \mathrm{~ms}$ & $5 \mathrm{~ms}$ \\
\hline$\tau_{A}^{0}$ & $0.4 \mathrm{~ms}$ & $0.4 \mathrm{~ms}$ \\
\hline$\tau_{A}^{\infty}$ & $30 \mathrm{~ms}$ & $30 \mathrm{~ms}$ \\
\hline$\tau_{L}^{0}$ & $30 \mu \mathrm{s}$ & $90 \mu \mathrm{s}$ \\
\hline$\tau_{L}^{\infty}$ & $1.4 \mathrm{~ms}$ & $4.3 \mathrm{~ms}$ \\
\hline$\tau_{T}^{0}$ & $100 \mu \mathrm{s}$ & $34 \mu \mathrm{s}$ \\
\hline$\tau_{T}^{\infty}$ & $1 \mathrm{~ms}$ & $340 \mu \mathrm{s}$ \\
\hline$\tau_{Q}^{0}$ & $4 \mu \mathrm{s}$ & $4 \mu \mathrm{s}$ \\
\hline$t_{c}$ & $20 \mathrm{~ms}$ & $60 \mathrm{~ms}$ \\
\hline$\tau_{Q}\left(t_{c}\right)$ & $1.4 \mathrm{~s}$ & $1.4 \mathrm{~s}$ \\
\hline
\end{tabular}

TABLE II: Values of the different time scales for two operating pressures.

where $\lambda_{i 0}=\sqrt{\epsilon_{0} k_{B} T_{i} / n_{0} e^{2}}$ is the initial ion Debye length.

It should be noted that the time scale for dust charge fluctuations strongly depends on plasma density and can vary from microsecond for initial stages of plasma decay up to seconds in case of almost extinguished plasma. Taking into account Eq.13 and Eq.21, the time dependence of $\tau_{Q}$ can be expressed as [20]:

$$
\tau_{Q}^{-1}=\frac{1}{\tau_{Q}^{0}} \exp \left(-t / \tau_{L}\right)
$$

To understand the dusty plasma decharging dynamics we have to compare different time scales. In Tab.II, time scales for this experiment are presented. It can be seen that the initial charge fluctuation time scale is the shortest. The temperature relaxation time scale is shorter or becomes comparable (for 0.4mbar) to the plasma density decay time scale, and plasma losses mainly determined by diffusion. The latest means that for our experimental conditions dust particles did not affect plasma decaying

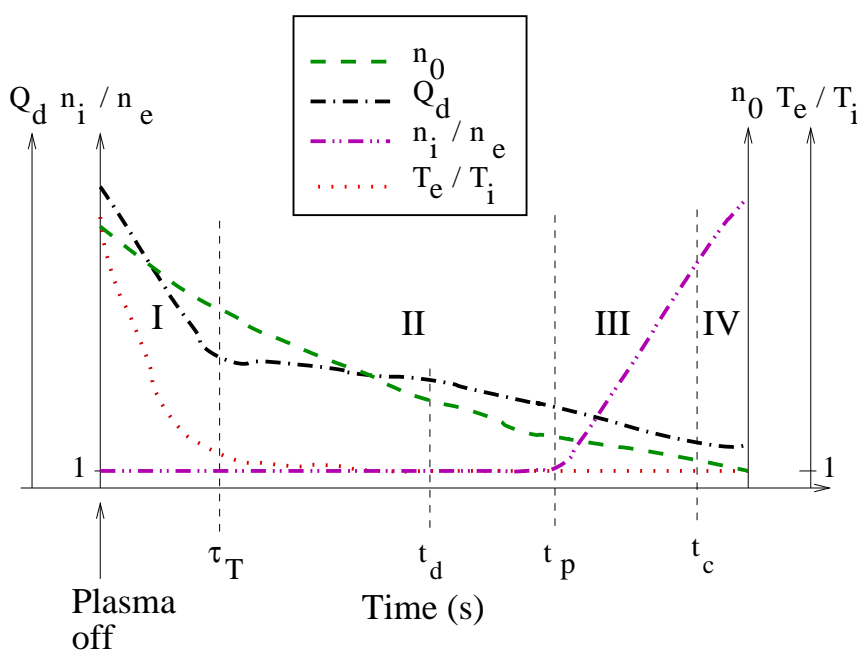

FIG. 7: (Color online) Qualitative time evolution of dust charge, plasma density and electron temperature during the afterglow Four stages of the dust plasma decay can be identified: I - temperature relaxation stage up to $t_{T}$ II - plasma density decay stage up $t_{p}$, III- dust charge volume stage $t_{c}$, IV - frozen stage. at initial stage.

The fig.7 presents the qualitative dependence of the main plasma and dust particle parameters during the afterglow. Four stages of the dust plasma decay can be labeled. As we can see the first stage of the plasma decay $\left(t<\tau_{T}\right)$ is characterized by the electron temperature $T_{e}$ drop down to the room temperature, while the plasma density (especially in case $\tau_{T}^{0}<\tau_{L}^{0}$ ) is slightly decreased. As the charging time scale is almost independent on $\tilde{T}_{e}$ (Eq.21), the charge is still determined by its equilibrium value (Eq.12). During the temperature relaxations stage the particle charge should decrease to the value [20]:

$$
Q_{r T}=\frac{1}{\tilde{T}_{e 0}}\left(\frac{\varphi_{e q}(1)}{\varphi_{e q}\left(\tilde{T}_{e 0}\right)}\right) Q_{0} \simeq \frac{Q_{0}}{62} \simeq-15 e
$$

where $Q_{0}$ is the initial dust charge in the plasma and $Q_{r T}$ the value of dust residual charge at the end of first decay stage. The dust charge in the plasma $Q_{0}$ was estimated as $Q_{0}=-950 e$ solving numerically the equation 19 , with given parameters $T_{i}=300 \mathrm{~K}$ and $T_{e} \simeq 3 \mathrm{eV}$, for argon plasma with $n_{i} \cong n_{e}$. At the next stage of decay, electron temperature is stabilized while the plasma density is still decreasing (see fig 7). So $\tau_{Q}$ continues increasing according to Eq.12 and Eq.21. When $\tau_{Q}$ becomes comparable to $\tau_{L}$, the particle charge can not be considered as in equilibrium and to determine particle charge we should use the Eq.12. The time scale when the particle charge starts sufficiently deviating from the equilibrium can be estimated as (Eq.15 and Eq.22):

$$
t_{d} \sim-\tau_{L}^{\infty} \ln \left(\frac{8}{3} \cdot\left(\frac{\lambda_{i 0}}{\Lambda}\right)^{2} \cdot \frac{l_{\text {in }}}{r_{d}}\right) \sim 6 \tau_{L}^{\infty}
$$

However, according to Eq.12, as long as plasma is neutral $\left(n_{e}=n_{i}\right)$ the charge on dust particle does not change. The plasma will keep quasineutrality until decaying rates for the electrons and ions are the same. It will be true in the case of ambipolar diffusion. When the nature of diffusion changes, electrons and ions start to diffuse independently that will lead to changing $n_{e} / n_{i}$ ratio and consequently the dust charge change.

The nature of the plasma diffusion changed when the particle volume charge can not be ignored or when the plasma screening length becomes comparable to the chamber size. In first case the ion diffusion will be influenced by the negatively charged dust particles, while the electrons will be free to go. In second case, large density differences appear over distances less than the screening length and electrons and ions diffuse independantly. Lets us estimate the characteristic times for both cases.

The influence of the overall particle charge is determined by the value of Havnes parameter $P_{e}=-N Q_{d} / e n_{e}$. Based on the model discussed, the qualitative evolution of the Havnes parameter $P_{e}$ in dusty plasma afterglow can be plotted as shown in Fig.8. The initial value of $P_{e}$ is small ( $\sim 0.06$ with an estimated dust density $N \sim 2 \cdot 10^{5} \mathrm{~cm}^{-3}$ and $n_{0} \simeq n_{e 0} \sim 5 \cdot 10^{9} \mathrm{~cm}^{-3}$ ) and there is no influence of dust. At the first stage of decaying (temperature relaxation stage) $P_{e}$ decreases due to dramatic decrease of dust charge while the plasma density decrease of a factor 1.1. At $\tau_{T}, P_{e}$ reaches its minimum value about $1 \times 10^{-3}$. After this $P_{e}$ starts increasing. During this stage dust particle charge changes slowly while 


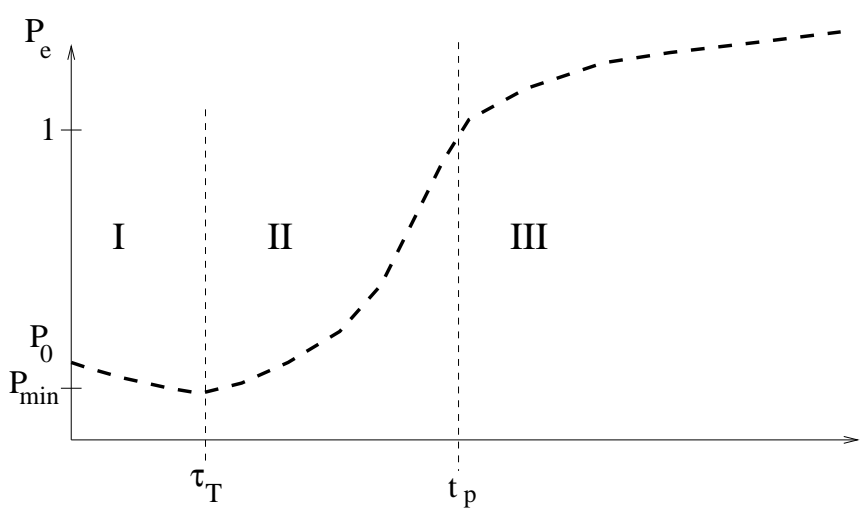

Time (s)

FIG. 8: Qualitative time evolution of the Havnes parameter after the power is switched off.

plasma number density decays fast (see Fig.7). The time at which $P_{e}$ becomes $\sim 1$ can be estimated as (Eqs.1318):

$$
t_{p} \sim \tau_{L}^{\infty} \ln \left(\left(\frac{T_{e 0}}{T_{n}}\right)\left(\frac{-e n_{0}}{Q_{d} N}\right)\right) \sim 8 \tau_{L}^{\infty}
$$

The screening length becomes comparable to the chamber size, i.e. $\lambda_{i}\left(\tilde{n}_{c}\right) \sim \Lambda$ when the density drops down to $\tilde{n}_{c}=\lambda_{i 0}^{2} / \Lambda^{2}$. This occurs at $[20]$ :

$$
t_{c} \sim \tau_{L}^{\infty} \ln \tilde{n}_{c}^{-1} \sim 10 \tau_{L}^{\infty}
$$

At time $\min \left[t_{p}, t_{c}\right]$, electrons start running away faster than ions and the ratio $n_{i} / n_{e}$ grows. For our experimental conditions $t_{p}<t_{c}$, thus the neutrality violation due to presence of dust particles happens before Debye length exceeds the chamber size. So the third stage of dusty plasma decay starts at $t_{p}$. During this stage the charge on dust particles is changed due to changing of $n_{e} / n_{i}$ ratio. At this stage $t_{d}<t_{p}$, thus the kinetic Eq.12 should be used for estimations of the charge variation. The upper limit of the charge change can be estimated ignoring the electron current and considering the time interval between $t_{p}$ and $t_{c}$,

$$
\begin{aligned}
\frac{d Q}{d t} & <\left.J_{i}\right|_{t_{p}} ^{t_{c}} \\
& <\left.\pi e r_{d}^{2} n_{i}\left(t_{p}\right) v_{T_{i}}\left(1-\frac{e}{4 \pi \epsilon_{0} k_{B} r_{d} T_{i}} Q_{d}\right)\right|_{t_{p}} ^{t_{c}}
\end{aligned}
$$

Solving Eq.28, the charge should evolve following:

$$
Q_{d}=\left(Q_{d T}-\frac{1}{\alpha}\right) \exp (-K \alpha \Delta t)+\frac{1}{\alpha}
$$

where $\alpha=e / 4 \pi \epsilon_{0} k_{B} r_{d} T_{i} \sim 0.28 / e, K=\pi e r_{d}^{2} n_{i}\left(t_{p}\right) v_{T_{i}} \sim$ $190 e$, and thus $(K \alpha)^{-1} \sim 20 m s \sim \Delta t=\left(t_{c}-t_{p}\right)$. Therefore, the charge during the third stage decreases to $-3 e$. At the forth stage of plasma decay, $t>t_{c}$, the plasma density decreases such that any further changes of dust charge become negligible and charge remains constant for a while. Thus the final residual charge for our condition is expected to be about $Q_{d_{\text {res }}} \sim-3 e$ which is

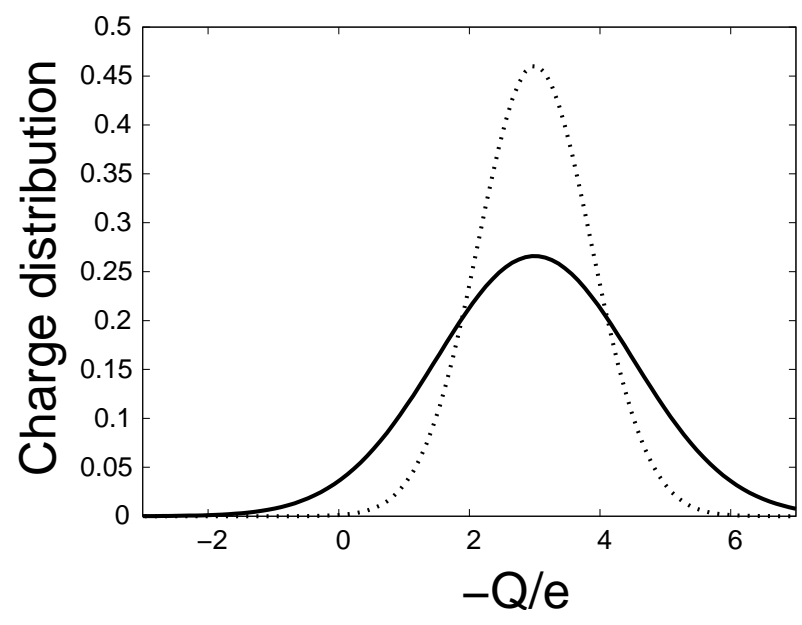

FIG. 9: Charge distribution with and without image force correction. Dotted line represents distribution with $\Delta Q=$ $0.5 \sqrt{Q}$. Solid line represents distribution with $\Delta Q=0.87 \sqrt{Q}$ $[37]$.

well correlated with the charges measured in the experiment (Tab.I).

The observation of neutral and positively charged particles in our experiments can be explained by the particle charge distribution. The charge distribution in dust particle ensemble is due to charge fluctuation on every individual dust particle. It has been shown [36-40] that rms of stochastic charge fluctuations varies as $\delta \cdot \sqrt{\left\langle Q_{d}\right\rangle}$, where $\delta$ is a parameter depending on plasma conditions and close to 0.5. Thus for mean charge around $-3 e$ the charge distribution should lay mainly between $-1 e$ and $-5 e$. However in our case we can expect broader distribution taking into account two reasons. First for small particles with a charge of about few electrons the image force (polarizability) correction starts playing role, see [37]. And for the electron temperature less then $0.1 \mathrm{eV}$ the correction factor can be up to 3 . That is significantly broadening distribution so dust particles can experience fluctuations to neutral and positive charges, see fig.9. Another reason for the broadened distribution could be the earlier freezing of the charge distribution so it remains the same while the mean charge decreases. Indeed as $t>t_{d}$ the charge is not in equilibrium with surrounding plasma so the existing charge distribution should remain unchanged or changed slightly during the later stages. Unfortunately, in this experiment the ratio between positively and negatively charged particles has not been measured due to experimental limitations concerned with the fact that dust grains do not stay in the laser sheet for a sufficiently long time due to their out of plane thermal motion. Thus to elaborate the true reason of the presence of residual charges of different polarities one need additional measurement of charge distributions.

The considered decharging model predicts a higher residual charge for lower pressure. For low pressure the temperature relaxation time scale exceeds plasma density decay time (see characteristics times for 0.4 mbar in Table II). This leads to quick increase of charging time and makes charge freezing at early stage of decay keeping charge at relatively high value. In fact bigger charges were measured for the higher pressure. A possible expla- 
nation for this is an electron re-heating in late afterglow from metastable atoms [41]. The presence of a significant amount of argon metastables in PKE discharge have been established by plasma modeling[42] and by experimental measurements[43]. Due to re-heating the electrons can have temperature few times more than the room temperature which will lead to the increase of residual charge on dust particles. As re-heating is more effective for highpressures thus bigger charges are expected.

\section{CONCLUSION}

Residual dust particle charges have been measured in late afterglow of a dusty plasma. Dust particles have been grown directly in the plasma by sputtering of a polymer material previously deposited on electrodes. Dust particles with a radius of few hundreds of nanometers are levitating after the power of the discharge is switched off. The gravity was balanced by an upward thermophoretic force. Residual charges were determined from analysis of dust oscillations, which were excited by applying sinusoidal bias to the bottom electrode. Positive, negative and non-charged dust particles have been detected. Mean residual charge for $200 \mathrm{~nm}$ radius particles was measured. The particle charge is about $-5 e$ at pressure of 1.2 mbar and about $-3 e$ at pressure of 0.4 mbar. A model for the dusty plasma decay was exploited to explain the experimental data. According to this model the dust plasma decay occurs in four stages: temperature relaxation stage, density decay stage, dust charge volume stage, and frozen stage (ice age IV). The main decrease of the dust charge happens during the first stage due to cooling of the electron gas. The final residual charge established during the third stage when the density of ions exceeds the density of electrons and the plasma density is still high enough to affect the charge. Measured values of the dust residual charges are in a good agreement with values predicted by the model. However the residual charge dependence on discharge conditions and detection of positively charged particles show that a more detailed model taking into account various phenomena (electron re-heating, electron release [44], afterglow chemistry) in decaying plasma have to be developed for better understanding of dusty plasma afterglow.

\section{Acknowledgments}

The authors would like to thank S. Dozias and B. Dumax for electronic support, J. Mathias for optical support and Y. Tessier for experimental support. The PKE-Nefedov chamber has been made available by the Max-Planck-Institute for Extraterrestrial Physics, Germany, under the funding of DLR/BMBF under grants No.50WM9852. This work was supported by CNES under contract $793 / 2000 / C N E S / 8344$. This paper is partially supported by the French-Australian integrated research program (FAST) of the French foreign affairs ministry and the International Science Linkages established under the Australian Government's innovation statement, Backing Australia's Ability'.
[1] A. P. Nefedov, G. E. Morfill, V. E. Fortov, H. M. Thomas, H. Rothermel, T. Hagl, A. V. Ivlev, M. Zuzic, B. A. Klumov, A. M. Lipaev, et al., New J. Phys. 5, 33.1 (2003).

[2] A. Bouchoule and L. Boufendi, Plasma Sources Sci. Technol. 2, 204 (1993).

[3] M. Cavarroc, M. C. Jouanny, K. Radouane, M. Mikikian, and L. Boufendi, J. Appl. Phys. 99, 064301 (2006).

[4] M. Mikikian, L. Boufendi, A. Bouchoule, H. M. Thomas, G. E. Morfill, A. P. Nefedov, V. E. Fortov, and the PKENefedov team, New J. Phys. 5, 19 (2003).

[5] M. Mikikian and L. Boufendi, Phys. Plasmas 11, 3733 (2004).

[6] D. Samsonov and J. Goree, J. Vac. Sci. Technol. A 17, 2835 (1999).

[7] A. A. Samarian and B. W. James, Phys. Lett. A 287, 125 (2001).

[8] A. Bouchoule, Dusty Plasmas: Physics, Chemistry and Technological impacts in Plasma Processing (Wiley, 1999).

[9] I. A. Belov, A. S. Ivanov, D. A. Ivanov, A. F. Pal, A. N. Starostin, A. V. Filippov, A. V. Demyanov, and Y. V. Petrushevich, JETP 90, 93 (2000).

[10] U. Konopka, F. Mokler, A. V. Ivlev, M. Kretschmer, G. E. Morfill, H. M. Thomas, H. Rothermel, V. E. Fortov, A. M. Lipaev, V. I. Molotkov, et al., New J. Phys. 7, 227 (2005).

[11] S. Vladimirov, K. Ostrikov, and A. Samarian, Physics and Applications of Complex Plasmas (Imperial Press, London, 2005).

[12] E. B. Tomme, D. A. Law, B. M. Annaratone, and J. E. Allen, Phys. Rev. Lett. 85, 2518 (2000).
[13] C. Zafiu, A. Melzer, and A. Piel, Phys. Rev. E 63, 066403 (2001).

[14] A. A. Samarian and S. V. Vladimirov, Phys. Rev. E 67, 66404 (2003).

[15] O. S. Vaulina, A. A. Samarian, B. W. James, O. F. Petrov, and V. E. Fortov, JETP 96, 1037 (2003).

[16] N. J. Prior, L. W. Mitchell, and A. A. Samarian, J. Phys. D: Appl. Phys. 36, 1249 (2003).

[17] S. Ratynskaia, S. Khrapak, A. Zobnin, M. H. Thoma, M. Kretschmer, A. Usachev, V. Yaroshenko, R. A. Quinn, G. E. Morfill, O. Petrov, et al., Phys. Rev. Lett. 93, 085001 (2004).

[18] C. Arnas, M. Mikikian, and F. Doveil, Phys. Rev. E 60, 7420 (1999).

[19] M. A. Childs and A. Gallagher, J. Appl. Phys. 87, 1086 (2000).

[20] A. Ivlev, M. Kretschmer, M. Zuzic, G. E. Morfill, H. Rothermel, H. Thomas, V. E. Fortov, V. I. Molotkov, A. P. Nefedov, A. Lipaev, et al., Phys. Rev. Lett. 90, 055003 (2003).

[21] H. Rothermel, T. Hagl, G. E. Morfill, M. H. Thoma, and H. M. Thomas, Phys. Rev. Lett. 89, 175001 (2002).

[22] P. Shukla and A. Mamun, Introduction to Dusty Plasma Physics (IOP Publishing, 2002).

[23] F. Zheng, Adv. Colloid \& Interf. Sci. 97, 255 (2002).

[24] R. N. Varney, Phys. Rev. 88, 362 (1952).

[25] Z. Li and H. Wang, Phys. Rev. E 70, 021205 (2004).

[26] I. Goldhirsch and D. Ronis, Phys. Rev. A 27, 1616 (1983).

[27] I. Goldhirsch and D. Ronis, Phys. Rev. A 27, 1635 (1983). 
[28] L. Waldmann, Z. Naturforsch. 14a, 589 (1959).

[29] K. DeBleecker, A. Bogaerts, and W. Goedheer, Phys. Rev. E 71, 066405 (2005).

[30] W. Li and E. J. Davis, J. Aerosol Sci. 26, 1063 (1995).

[31] W. Li and E. J. Davis, J. Aerosol Sci. 26, 1085 (1995).

[32] M. A. Gallis, D. J. Rader, and J. R. Torczynski, Aerosol Sci. \& Technol. 36, 1099 (2002).

[33] L. Landau and E. Lifchitz, Physique Théorique, Tome 1 (Édition Mir Moscou, 1982).

[34] Y. P. Raizer, Gas Disharge Physics (Springer, Berlin, 1991).

[35] V. N. Tsytovich, Phys. Usp. 40, 53 (1997).

[36] T. Matsoukas and M. Russell, J. Appl. Phys. 77, 4285 (1995).

[37] T. Matsoukas, M. Russell, and M. Smith, J. Vac. Sci. Technol. A 14, 624 (1996).

[38] O. S. Vaulina, S. A. Khrapak, A. P. Nefedov, and O. F.
Petrov, Phys. Rev. E 60, 5959 (1999).

[39] S. A. Khrapak, A. P. Nefedov, O. F. Petrov, and O. S. Vaulina, Phys. Rev. E 59, 6017 (1999).

[40] C. Cui and J. Goree, IEEE Trans. Plasma Sci. 22, 151 (1994).

[41] G. Wenig, M. Schulze, P. Awakowicz, and A. v. Keudell, Plasma Sources Sci. Technol. 15, S35 (2006).

[42] M. R. Akdim and W. J. Goedheer, Phys. Rev. E 67, 066407 (2003).

[43] M. Mikikian, L. Boufendi, and A. Bouchoule, in Proc. $30^{\text {th }}$ EPS Conf. on Contr. Fusion and Plasma Phys., St Petersburg, edited by R. Koch and S. Lebedev (2003), vol. 27A of ECA, pp. O-3.1B.

[44] J. Berndt, E. Kovacevic, V. Selenin, I. Stefanovic, and J. Winter, Plasma Sources Sci. Technol. 15, 18 (2006). 